\title{
Efectividad de un programa de ejercicios diseñado para personas con osteoporosis y osteopenia en el manejo del dolor y la calidad de vida
}

\author{
María José Ponce Darós, Nuria Sempere Rubio y Sara Cortés Amador \\ Universidad de Valencia (España)
}

\begin{abstract}
Objetivo: Estudiar si un programa de ejercicio diseñado para pacientes con osteoporosis y osteopenia puede disminuir el dolor y mejorar la calidad de vida. Método: Han participado 84 pacientes entre 50 y 83 años, la mitad en el grupo experimental y el resto, en el control. El estudio se realizó en el CEAM de Puçol (Valencia) durante 9 meses, en los que el grupo experimental realizaba los ejercicios supervisados por la fisioterapeuta 2 días a la semana durante 1 hora. En este periodo de tiempo, se midió el dolor, calidad de vida, asistencia y satisfacción con el servicio. Resultados: Tras realizar prueba $t$ de muestras relacionadas, se observa como el grupo experimental disminuye su dolor y mejora su calidad de vida de la valoración inicial a la final. Por el contrario, el grupo control empeora. Tras realizar pruebas de regresión, se encuentra relación estadísticamente significativa entre el dolor, calidad de vida y asistencia al programa de ejercicios. La satisfacción percibida por los pacientes del grupo experimental fue muy buena, y al 100\% de ellos les gustaría poder seguir realizando el programa de ejercicios en el futuro. Conclusiones: La realización de un programa de ejercicio físico específico para pacientes con osteoporosis y osteopenia, supervisado por un fisioterapeuta, disminuye el dolor y mejora la calidad de vida de los participantes.
\end{abstract}

Palabras clave: Osteoporosis, osteopenia, programa ejercicio, dolor, calidad de vida.

Effectiveness of an exercise program designed for persons with osteoporosis and osteopenia on pain management and quality of life. Objective: To study if an exercise program designed for patients with osteoporosis and osteopenia can be decrease the pain and improve quality of life. Method: 84 patients between 50 and 83 years old was participated at the study, 42 persons in the experimental group and 42 persons in the control group. The study was made in Puçol CEAM (Valencia) for 9 months on the experimental group was performed exercises supervised by the physical therapist 2 days a week for 1 hour. In this period of time, pain, quality of life, support and service satisfaction was measured. Results: After performing paired samples t test, shows how the experimental group decreased their pain and improve their quality of life from the initial to the final score. By contrast, the control group worsened. After testing regression is statistically significant relationship between pain, quality of life and assistance exercises. The perceived satisfaction by patients in the experimental group was very good, and $100 \%$ of them would like to continue doing the exercise program. Conclusions: The implementation of a specific exercise program for patients with osteoporosis and osteopenia reduces pain and improves quality of life of participants.

Keywords: Osteoporosis, osteopenia, exercise program, pain, quality of life.

Correspondencia: María José Ponce Darós. Departamento de Fisioterapia. Facultad de Fisioterapia. Universidad de Valencia. C/ Gascó Oliag, 5. C.P.: 46010. Valencia (España). E-mail: Maria.Jose.Ponce@uv.es 
Se sabe que la osteoporosis es una enfermedad generalizada del sistema esquelético, caracterizada por una disminución de la masa ósea y por el deterioro micro-arquitectónico del tejido óseo cortical y sobre todo, trabecular, que aumenta la fragilidad del hueso y disminuye su resistencia (densidad y calidad ósea). Tiene lugar cuando la cantidad de hueso que se reabsorbe es superior al que se forma. Este desequilibrio origina la pérdida progresiva de masa ósea y el deterioro de su estructura. La consecuencia es una fragilidad esquelética con tendencia a padecer fracturas (II Plan para la Prevención y Control de la Osteoporosis en la Comunitat Valenciana, 2009; Pérez et al., 2011).

La osteoporosis se ha convertido en una auténtica "epidemia del siglo XXI", ya que es una enfermedad que afecta a la población a nivel mundial, presentándola en la actualidad más de 200 millones de personas. Se calcula que en un futuro del 30 al 50\% de las mujeres postmenopáusicas la padecerán (Schurman et al., 2013).

En España unos 3,5 millones de personas están diagnosticadas de osteoporosis, siendo las mujeres las más perjudicadas (2,5 millones de mujeres y 1 millón de hombres). En concreto, el 35 por ciento de las mujeres sufre la enfermedad a partir de los 50 años, un porcentaje que aumenta hasta el 52 por ciento en las mujeres mayores de 70 años (SEIOMM, 2013).

El impacto económico de la osteoporosis es realmente importante, desde la prevención de las fracturas, el tratamiento de las mismas y hasta las repercusiones posteriores a las fracturas hacen que la enfermedad tenga un elevado impacto sobre el presupuesto global sanitario. A parte de los gastos derivados de las ventas de productos farmacéuticos para la osteoporosis, los datos epidemiológicos y las cifras de las diferentes fracturas, sobre todo las de cadera, hacen que se considere a la osteoporosis como un problema socio-sanitario. Aunque si bien la osteoporosis no suele poner en peligro la vida de las personas, las fracturas por fragilidad causadas por ella son responsables de una elevada mortalidad, morbilidad, dolor crónico, discapacidad, ingreso en instituciones y costes económicos para los pacientes y sus familiares. Desde este punto de vista supone una considerable carga económica y de atención para los servicios sanitarios y sociales (II Plan para la Prevención y Control de la Osteoporosis en la Comunitat Valenciana, 2009).

La información aportada por la International Osteoporosis Foundation y la Federación Europea de Industrias y Asociaciones Farmacéuticas (EFPIA) para 2010, indica que esta enfermedad genera unos gastos de 37.000 millones de euros a nivel europeo que, unidos a la estimación de los años vividos con la calidad de vida de los mismos, provocaron que en 2010 los gastos ascendieran a 99.000 millones de euros. Además, se estima que en 2025 los gastos aumenten a 121.000 millones, lo que implica un 22 por ciento más (SEIOMM, 2013). Con intención de disminuir la factura 
económica y mejorar la calidad de vida de los pacientes, la prevención de la osteoporosis juega un papel imprescindible.

La prevención debe ser el aspecto fundamental en el control de la osteoporosis, convirtiéndose en el objetivo prioritario de los equipo de atención primaria. Para ello se han de tener en cuenta los factores de riesgo de padecer osteoporosis para intentar modificarlos y/o incidir sobre ellos. De todos los factores de riesgo, el sexo y la edad son los dos más importantes para sufrir osteoporosis/osteopenia, por ello, se tendrá que tener una mayor precaución y énfasis en la prevención en las mujeres y en las personas mayores.

Para prevenir la osteoporosis en la actualidad, existen diversos procedimientos o actuaciones, que se pueden agrupar del siguiente modo:

Evitar y controlar los posibles factores de riesgo, seguir una dieta equilibrada y saludable, y farmacología para aumentar la DMO en el caso que sea necesario.

Realizar programas de ejercicio físico moderado adaptado a las características y necesidades de cada individuo.

Programas de educación sanitaria acerca de la osteoporosis.

Otras líneas de actuación multidisciplinares y multifactoriales para prevenir caídas.

En consecuencia, la prevención de caídas es sumamente importante en las personas mayores, tanto por los efectos que pueden acarrear como lesiones físicas (fracturas, dolor, deterioro en la movilidad, menor funcionalidad e incapacidad, dependencia de terceros) y por afectar negativamente a la calidad de vida. Además, las personas que se caen mucho tienen un miedo considerable a volver a caerse, y esta circunstancia, les puede provocar que se muevan menos debido al miedo, por lo que se vuelven más sedentarios e inactivos, con todos los problemas que vienen derivados de esa inactividad. Diversos estudios demuestran que el miedo a caerse tiene unas consecuencias negativas en la funcionalidad, en la sensación subjetiva de bienestar y en la consiguiente pérdida de autonomía; y a su vez, el deterioro funcional, físico o de la calidad de vida está claramente relacionado con el miedo a caerse. Para ello, se han recomendado múltiples intervenciones para prevenir caídas en las personas mayores, conllevando a cambios que resultan en reforzar su confianza para realizar las actividades (Alcalde, 2010; Lázaro, 2009; Lázaro 2008; Párraga et al., 2010).

Por tanto, el ejercicio físico será un pilar fundamental en la prevención y tratamiento de la osteoporosis/osteopenia, ya que puede ayudar a aumentar la DMO de los pacientes, o al menos, disminuir la pérdida progresiva debida a la edad, y por otro lado, puede disminuir caídas (al mejorar el equilibrio, coordinación y estado físico en general); disminuir el miedo a las caídas al sentirse más seguro, disminuir el dolor y mejorar la calidad de vida de los participantes. 
En la actualidad se sabe que el ejercicio físico ayuda muy positivamente a llevar una vida saludable y a envejecer con una mejor calidad de vida, por eso la OMS recomienda la práctica de ejercicio físico en todas las edades, y pone especial énfasis en las personas mayores, promoviendo el "envejecimiento activo" para mejorar la calidad de vida de las personas a medida que envejecen, favoreciendo sus oportunidades de desarrollo para una vida saludable, participativa y segura (Organización Mundial de la Salud, 2002; Sociedad Española de Geriatría y Gerontología, 2012).

\section{Hipótesis y objetivos}

Hipótesis: La realización continuada en el tiempo de un programa específico de ejercicio físico, supervisado por un/a fisioterapeuta, disminuirá el dolor y mejorará la calidad de vida de los participantes.

Objetivos:

Objetivo general: Estudiar si un programa de ejercicio diseñado para pacientes con osteoporosis y osteopenia puede disminuir el dolor y mejorar la calidad de vida de los participantes.

Objetivos específicos:

Disminuir el dolor (que se valorará mediante la Escala Verbal Numérica (EVN) numerada del 0 al 10).

Mejorar la calidad de vida (que se valorará mediante la escala de calidad de vida para la osteoporosis ECOS-16).

Valorar la asistencia presencial por los participantes al programa de ejercicios para saber si los resultados obtenidos en las diferentes escalas han tenido que ver con ésta.

Valorar la satisfacción percibida por los participantes acerca del programa de ejercicios, como indicador de calidad del servicio prestado.

\section{MÉTODO}

\section{Participantes}

Se seleccionó un total de 84 pacientes entre 50 y 83 años de edad, con los siguientes criterios de inclusión y exclusión:

Criterios de inclusión:

Edad de 50 a 83 años.

Con diagnóstico de osteoporosis u osteopenia.

Que no necesiten ayuda para deambular, o que utilicen sólo bastón o muleta.

Firmar consentimiento informado.

Vivir en comunidad (no institucionalizados). 
Criterios de exclusión:

Que sean personas con dependencia alta o moderada.

Que utilicen andador o silla de ruedas.

Que padezcan deterioro cognitivo moderado o grave.

Que padezcan enfermedades cardíacas graves.

Que hayan padecido un ACV que les haya dejado secuelas de hemiplejía.

Que hayan sufrido una fractura reciente, de la cual todavía no se han recuperado.

Tras la selección de la muestra, 42 sujetos formaron parte del grupo experimental (GE) y otros 42, del grupo control (GC).

\section{Instrumentos}

El material necesario para el estudio fue el siguiente:

El programa de ejercicios se realizó en el gimnasio del CEAM donde se llevó a cabo el proyecto, y se utilizaron pelotas, pesas, bandas elásticas y sillas.

La recogida de la información necesaria se llevó a cabo a través de:

Informe de los/las médicos/as de atención primaria para confirmar el diagnóstico de los pacientes (osteoporosis/osteopenia) y los criterios de inclusión y exclusión).

Hojas de consentimiento informado en las que se refleje que los sujetos de ambos grupos están dispuestos a participar en este proyecto.

Escalas de valoración y encuestas personales para ambos grupos:

Cuestionario sobre datos sociodemográficos y sanitarios.

Escala de calidad de vida para la osteoporosis ECOS-16 (oscila de 16 a 80 puntos; a mayor puntuación, peor calidad de vida).

Escala para medir el dolor EVA numerada o EVN (medida de 0 a 10; a mayor puntuación, mayor dolor).

Registro de asistencia al programa de ejercicios por parte del GE.

\section{Procedimiento}

Este estudio se realizó en el CEAM de Puçol (Valencia) durante 9 meses, en los que el GE realizaba los ejercicios supervisados por la fisioterapeuta 2 días a la semana durante 1 hora. El GC no realizaba el programa de ejercicios, pero a ambos grupos se les midió el dolor y la calidad de vida al inicio $\left(0^{\circ} \mathrm{mes}\right)$ y al finalizar el estudio ( $10^{\circ}$ mes). En la valoración final también se midió la asistencia y la satisfacción con el servicio en los sujetos del GE.

El programa realizado por el GE se basaba en ejercicios de miembros superiores (MMSS), miembros inferiores (MMII), tronco y cuello, no centrándose en una parte específica del cuerpo, sino realizando un ejercicio global. Los ejercicios se han 
practicado en grupo para producir cierto compromiso y resultar más divertidos para los participantes y conseguir una mayor adhesión al programa. Cada sesión se dividió en 7 partes: $1^{\text {a }}$ : calentamiento en sedestación (ejercicios suaves con y sin pelota); $2^{a}$ : ejercicios en bipedestación I (ejercicios de movilidad, coordinación y flexibilidad); $3^{\mathrm{a}}$ : ejercicios con pesas; $4^{\mathrm{a}}$ : ejercicios en bipedestación II (ejercicios de equilibrio); $5^{\text {a }}$ : estiramientos globales de MMII; $6^{\text {a }}$ : ejercicios con bandas elásticas (MMSS y MMII); $7^{\text {a }}$ : vuelta a la calma (estiramientos MMSS, cuello y ejercicios de relajación).

Con este trabajo se pretende estudiar si tras realizar el programa de ejercicios, el GE presenta alguna diferencia significativa con el GC, el cual no realizará este programa.

\section{RESULTADOS}

Los análisis estadísticos se han realizado mediante el programa de estadística PASW 19.0 (SPSS Inc.), con licencia de la Universidad de Valencia. Se ha estimado el efecto del protocolo de ejercicio y su intervalo de confianza al $95 \%$. Se ha considerado como nivel de significación estadística una $p<0.05$.

Tabla 1. Estadísticos descriptivos de las variables "Género", "Edad” y "Diagnóstico" en función del grupo

\begin{tabular}{|c|c|c|}
\hline & Grupo experimental & Grupo control \\
\hline$N$ & 42 & 42 \\
\hline \multirow{2}{*}{ Género } & Mujeres: $39(92.9 \%)$ & Mujeres: $41(97.6 \%)$ \\
\hline & Hombres: $3(7.1 \%)$ & Hombres: $1(2.4 \%)$ \\
\hline \multirow{5}{*}{ Edad } & Media $=67.81$ años $\pm 6.7 S D$ & Media $=65.57$ años $\pm 9.89 S D$ \\
\hline & Mín.=53 años & Mín.=50 años \\
\hline & Máx.=81 años & Máx.=83 años \\
\hline & Media edad osteoporosis $=68.92 \pm 7.10 \mathrm{SD}$ & Media edad osteoporosis $=67.75 \pm 10.54 S D$ \\
\hline & Media edad osteopenia $=65.57 \pm 5.30 S D$ & Media edad osteopenia $=61.21 \pm 6.86 S D$ \\
\hline \multirow{2}{*}{ Diagnóstico } & Osteoporosis: $28(66.7 \%)$ & Osteoporosis: $28(66.7 \%)$ \\
\hline & Osteopenia: $14(33.3 \%)$ & Osteopenia: $14(33.3 \%)$ \\
\hline
\end{tabular}

Se realizó un análisis descriptivo de las variables cualitativas (obteniendo la frecuencia absoluta y porcentual) y de las cuantitativas (estadísticos de tendencia central, variabilidad y pruebas de normalidad).

Como se puede observar en la tabla 1, ambos grupos parten de características similares en la valoración inicial.

Por otro lado, se observa como en la valoración inicial (VI) ambos grupos presentan características similares respecto a la calidad de vida y dolor, teniendo mejor calidad de vida y menor dolor el GC. Pero en la valoración final (VF), el GE mejora su puntuación respecto a la calidad de vida y al dolor, y en cambio el GC empeora. 
PONCE, SEMPERE y CORTÉS. Efectividad de un programa de ejercicios en osteoporosis y osteopenia

Tabla 2. Estadísticos descriptivos de las variables "Calidad de vida" y "Dolor", en la valoración inicial y en la valoración final, en función del grupo

\begin{tabular}{lccc}
\hline & & Grupo experimental & Grupo control \\
\hline \multirow{2}{*}{ Calidad de vida (ECOS-16) } & VI & Media=40.66 $\pm 12.57 S D$ & Media=36.67 $\pm 12.01 S D$ \\
\cline { 2 - 4 } & VF & Media=29.47 $\pm 9.87 S D$ & Media=48.88 $\pm 13.56 S D$ \\
\hline \multirow{2}{*}{ Dolor (EVN) } & VI & Media=5.62 $\pm 2.29 S D$ & Media=4.67 $\pm 2.21 S D$ \\
\cline { 2 - 4 } & VF & Media=3.69 $\pm 3.05 S D$ & Media=6.62 $\pm 2.39 S D$ \\
\hline
\end{tabular}

Tabla 3. Estadísticos descriptivos "asistencia al programa de ejercicios por el grupo experimental"

\begin{tabular}{lc}
\hline & GRUPO EXPERIMENTAL \\
\hline $\begin{array}{l}\text { Asistencia al programa de } \\
\text { ejercicios (expresado en } \\
\text { porcentaje) }\end{array}$ & $\begin{array}{c}\text { Media=72.93\% } \% 25.23 S D \\
\text { Mín. }=14 \% \\
\text { Máx.=98\% }\end{array}$ \\
\hline & $\begin{array}{c}\text { Ningún problema: } 29 \text { personas que no han tenido problemas para no asistir, y por } \\
\text { tanto, su asistencia ha sido buena (69\%). }\end{array}$ \\
Motivos no asistencia & $\begin{array}{c}\text { Personas que han tenido problemas: } 13(31 \%) \text {. Motivos: problemas familiares: } 4 \\
(9.5 \%) ; \text { problemas de salud derivados de otra patología (ni osteoporosis ni osteopenia): } \\
9(21.4 \%) ; \text { no me gusta el programa de ejercicios: } 0(0 \%) ; \text { otros motivos: } 0(0 \%) .\end{array}$ \\
\hline $\begin{array}{l}\text { ¿Te gustaría poder seguir } \\
\text { realizando este programa } \\
\text { de ejercicios en un futuro? }\end{array}$ & Sí: 48 personas (100\%); No: $0(0 \%) ;$ No lo sé: $0(0 \%)$. \\
\hline
\end{tabular}

Tabla 4. Prueba $t$ de Student de muestras independientes entre la calidad de vida y el dolor medido en la valoración inicial y el grupo (control / experimental)

\begin{tabular}{lcc}
\hline Prueba $t$ de Student: variables a relacionar & Estadístico de contraste & ¿Hay relación estadísticamente significativa? \\
\hline $\begin{array}{l}\text { Calidad de vida inicial } \\
\text { Grupo (GC/GE) }\end{array}$ & \multirow{2}{*}{$t=1.491$} & No $(p=0.140)$ \\
\hline $\begin{array}{l}\text { Dolor inicial } \\
\text { Grupo }(\mathrm{GC} / \mathrm{GE})\end{array}$ & \multirow{2}{*}{$t=1.935$} & Media GE: $40.67 ;$ Media GC: 36.67 \\
\hline
\end{tabular}

Tabla 5. Prueba $t$ de Student de muestras independientes entre la calidad de vida y el dolor medido en la valoración final y el grupo (control/ experimental)

\begin{tabular}{lcc}
\hline Prueba $t$ de Student: variables a relacionar & Estadístico de contraste & ¿Hay relación estadísticamente significativa? \\
\hline $\begin{array}{l}\text { Calidad de vida final } \\
\text { Grupo (GC/GE) }\end{array}$ & $t=-6.336$ & Sí $(p=0.000)$ \\
& & Media GE: $29.47 ;$ Media GC: 45.88 \\
\hline Dolor final & & Tamaño efecto $(d)=1.38$ \\
Grupo (GC/GE) & $t=-4.901$ & Sí $(p=0.000)$ \\
& & Media GE: $3.69 ;$ Media GC: 6.62 \\
\hline
\end{tabular}

También se valoró la asistencia al programa de ejercicios por parte del GE. Se puede observar en la tabla 3 como la asistencia fue buena, con una media de asistencia de un $72.93 \%$ del total de las clases (2 días a la semana durante 9 meses). Los motivos que reflejaron las 13 personas que asistieron menos fueron problemas familiares y problemas derivados de otra patología; es decir, nadie señaló que asistiera menos o dejara de asistir porque no le gustara el programa de ejercicios. Además, al $100 \%$ de los sujetos del GE confirmaron que les gustaría poder seguir realizando el programa de ejercicios en el futuro. 
Para saber si ha habido diferencias estadísticamente significativas entre las medias de calidad de vida y de dolor en los sujetos del GC y GE, y saber si el programa de ejercicios ha sido efectivo, se han realizado diversas pruebas de estadística inferencial. En los estadísticos descriptivos realizados previamente, se observó cómo el GC tenía menos dolor y mejor calidad de vida en la valoración inicial que el GE; pero tras realizar la prueba $t$ de muestras independientes se observó que esas diferencias no eran estadísticamente significativas $(p>0.05)$; así que, tanto GC como GE partían de características similares al inicio del estudio. Sin embargo, tras realizar la prueba $t$ de muestras independientes de la valoración final, vemos cómo en ese momento sí existen diferencias estadísticamente significativas entre las medias de dolor y calidad de vida de ambos grupos, teniendo menos dolor y mejor calidad de vida el GE $(p<0.05)$.

Por otro lado, también se realizaron pruebas $\mathrm{T}$ de muestras relacionadas, en las que se pudo observar cómo el GE disminuye su dolor y mejora su calidad de vida de la valoración inicial a la final de manera estadísticamente significativa $(p<0.05)$. Por el contrario, el GC empeora de la valoración inicial a la final $(p<0.05)$.

\begin{tabular}{|c|c|c|}
\hline Prueba $t$ de muestras relacionadas: & Estadístico de contraste & ¿Hay relación estadísticamente significativa? \\
\hline \multirow{3}{*}{ Calidad de vida inicial y final (GE) } & \multirow{3}{*}{$t=7.121$} & Sí $(p=0.000)$ \\
\hline & & Media inicial: 40.66; Media final: 29.47 \\
\hline & & Tamaño del efecto $(d)=1.09$ \\
\hline \multirow{3}{*}{ Dolor inicial y final (GE) } & \multirow{3}{*}{$t=4.414$} & Sí $(p=0.000)$ \\
\hline & & Media inicial: 5.62; Media final: 3.69 \\
\hline & & Tamaño del efecto $(d)=0.68$ \\
\hline \multirow{3}{*}{ Calidad de vida inicial y final (GC) } & \multirow{3}{*}{$t=-6.036$} & Sí $(p=0.000)$ \\
\hline & & Media inicial: 36.67 ; Media final: 48.88 \\
\hline & & Tamaño del efecto $(d)=0.93$ \\
\hline \multirow{3}{*}{ Dolor inicial y final (GC) } & \multirow{3}{*}{$t=-8.188$} & Sí $(p=0.000)$ \\
\hline & & Media inicial: 4.67; Media final: 6.62 \\
\hline & & Tamaño del efecto $(d)=1.26$ \\
\hline
\end{tabular}

Para conocer qué variables influían respecto a otras, se realizaron pruebas de regresión simple, en las que se encontró relación estadísticamente significativa entre el dolor, calidad de vida y asistencia al programa de ejercicios:

A menor dolor, mejor calidad de vida $\left(F=17.882 ; R=0.556 ; R^{2}=0.309\right.$; $p<0.05)$. A mayor asistencia al programa de ejercicios, menor dolor $(F=18.365$; $\left.R=-0.561 ; R^{2}=0.315 ; p<0.05\right)$. A mayor asistencia al programa de ejercicios, mejor calidad de vida $\left(F=14.223 ; R=-0.512 ; R^{2}=0.262 ; p<0.05\right)$.

\section{DISCUSIÓN}

En la mayoría de estudios que realizan un programa de ejercicios para prevenir caídas en pacientes con osteoporosis y/u osteopenia, se ha demostrado que los 
ejercicios más beneficiosos son aquellos en los que se trabaja el equilibrio estático y dinámico, fuerza (sobre todo de MMII), flexibilidad, estiramientos y ejercicios de alto impacto que venzan la fuerza de la gravedad. Los autores recomiendan que para obtener más beneficios se realicen al menos 2 sesiones a la semana, y una duración mínima de tres meses. Cada sesión debe tener tres grandes bloques de ejercicios: empezar un calentamiento suave para pasar de manera progresiva a los ejercicios principales del programa de ejercicios (movilidad, velocidad, resistencia, fuerza y equilibrio), y volver a la calma con ejercicios de enfriamiento, estiramientos, relajación y ejercicios respiratorios (Kemmler et al., 2012; Bolton et al., 2012; Madureira et al., 2010; Orozco, 2012; Bergland, 2012; Wayne et al., 2012; Küçükçakır et al., 2013; Stolzenberg, 2013; Nutland et al., 2013), como se ha seguido en el presente trabajo.

Además de prevenir caídas, disminuir el dolor es un objetivo muy importante en este tipo de pacientes, ya que el dolor crónico es un factor de riesgo de caídas en la población anciana. El dolor crónico en la osteoporosis también se asocia con síntomas de depresión, ansiedad, frustración y aislamiento social (Madureira et al., 2010). En el presente estudio se disminuye el dolor gracias al programa de ejercicios, y de esta manera también se mejora la calidad de vida. Pero el dolor es una variable poco medida en la mayoría de estudios de prevención de caídas para pacientes con osteoporosis y osteopenia, por lo que se cree que debería de incluirse como variable a valorar en todos los estudios de prevención de caídas en personas mayores. En la bibliografía revisada sobre programas de prevención de caídas en pacientes con osteoporosis/osteopenia sólo se encontró un estudio en el que se valorara el dolor (Küçükçakır et al., 2013). En dicho trabajo lo midieron también con la escala de EVN/EVA.

En cambio, la calidad de vida es una variable más valorada. En el presente estudio ha sido medida con la escala de calidad de vida para pacientes con osteoporosis/osteopenia ECOS-16. En otros trabajos se valoró con la escala QUALEFFO-41 (Küçükçakır et al., 2013; Schröder et al., 2012). El resto de estudios la midieron con la escala de calidad de vida SF-36 (Saiz, 2011; Küçükçakır et al., 2013; Saucedo, 2009; Gianoudis et al., 2012), EuroQol-SD (Saiz, 2011), OPAQ (Madureira et al., 2010) y HRQOL (Bergland et al., 2012).

En el presente estudio, los pacientes del GE han disminuido su dolor y mejorado su calidad de vida gracias a la realización del programa de ejercicios. Además, la satisfacción percibida por los participantes ha sido muy buena, porque el $100 \%$ de los sujetos señala en la encuesta que le gustaría poder seguir realizando esta actividad en el futuro debido a los beneficios y mejoras adquiridas a nivel físico, funcional y psicológico, y también a nivel social, ya que al realizar el ejercicio en grupo se crea un vínculo entre los participantes y ven el programa de ejercicios no sólo como un tratamiento, sino como una actividad lúdica y social. 
La realización de un programa de ejercicio físico específico para pacientes con osteoporosis y osteopenia, supervisado por un fisioterapeuta, disminuye el dolor y mejora la calidad de vida de los participantes.

\section{REFERENCIAS}

Alcalde, P. (2010). Revisión: Miedo a caerse. Revista Española de Geriatría y Gerontología, 45(1), 38-44.

Bergland, A. (2012). Effect of exercise on falls efficacy in osteoporotic women with a history of vertebral fracture: A randomized, controlled trial. En: 8th Congress of the EUGMS. European Geriatric Medicine, 3, 33-143.

Bolton, K.L., Egerton, T., Wark, J., Wee, E., Matthews, B., Kelly, A., Craven, R., Kantor y S., Bennell, K.L. (2012). Effects of exercise on bone density and falls risk factors in postmenopausal women with osteopenia: a randomized controlled trial. Journal of science and medicine in sport. Sports Medicine Australia, 15(2), 102-109.

Gianoudis, J., Bailey, C.A., Sanders, K.M., Nowson, C.A., Hill, K., Ebeling, P.T. y Daly, R.M. (2012). 'Osteo-cise: Strong Bones for Life': Protocol for a Community-based Randomized Controlled Trial of a Multi-modal Exercise and Osteoporosis. Education Program for Older Adults at Risk of Falls and Fractures. BMC Musculoskeletal Disorders, 13, 78. DOI: 10.1186/1471-2474-13-78.

II Plan para la Prevención y Control de la Osteoporosis en la Comunitat Valenciana 2009-2013. (2009). Generalitat Valenciana. Conselleria de Sanitat.

Kemmler, W., Von Stengel, S., Bebenek, M., Engelke, K., Hentschke, C. y Kalender, W.A. (2012). Exercise and fractures in postmenopausal women: 12-year results of the Erlangen Fitness and Osteoporosis Prevention Study (EFOPS). Osteoporosis International, 23, 1267-1276. DOI: 10.1007/s00198-011-1663-5.

Küçükçakır, N., Altan, L. y Korkmaz, N. (2013). Effects of Pilates exercises on pain, functional status and quality of life in women with postmenopausal osteoporosis. Journal of Bodywork \& Movement Therapies, 17, 204-211.

Lázaro del Nogal, M., González-Ramírez, A. y González, E. (2008). Caídas y osteoporosis. Revista Española de Geriatría y Gerontología, 43(5), 330-333.

Lázaro del Nogal, M. (2009). Diagnóstico y tratamiento. Caídas en el anciano. Medicina Clínica, 133(4), 147-153.

Madureira, M.M., Bonfá, E., Takayama, L. y Pereira, R.M.R. (2010). A 12-month randomized controlled trial of balance training in elderly women with osteoporosis: Improvement of quality of life. Maturitas, 66, 206-211.

Nutland, H., Dean, A. y Laxminarayan, R. (2013). Use of wii fit in balance training in osteoporosis. Rheumatology, 52(1), 106.

Organización Mundial de la Salud (2002). Envejecimiento activo: un marco político. Revista Española de Geriatría y Gerontología, 37(2), 74-105.

Párraga, I., Navarro, B., Andrés, F., Denia, J.N., Elicegui, R.P. y López-Torres, J. (2010). Miedo a las caídas en las personas mayores no institucionalizadas. Gaceta Sanitaria, 24(6), 453459.

Pérez, L., Alonso, A., Roig, D., García, A., Guañabens, N., Peris, P., Torrijos, A., Beltrán, C.H., Fiter, J., Arboleya, L., Graña, J., Carbonell, J., Miquel, J., Holgado, S., Salas, E., Zubieta, J., Del Pino, J., Blanch, J., Caamaño, M., Rodríguez, M., Castañeda, S., Cerdá, D., Gómez, C., Calvo, J., Ciria, M. y Loza, E. (2011). Actualización 2011 del consenso 
Sociedad Española de Reumatología de osteoporosis. Reumatología Clínica, 7(6), 357379.

Saiz, J.R. (2011). Impacto de un programa de fisioterapia sobre la movilidad, el equilibrio y la calidad de vida de las personas mayores (Tesis doctoral inédita). Departamento de Medicina, Dermatología y Toxicología. Universidad de Valladolid.

Saucedo, P., Abellán, J., Gómez, P., Leal, M., Ortega, E., Colado, J.C. y Sainz de Baranda, P. (2009). Efectos de un programa de ejercicio físico sobre la calidad de vida en la postmenopausia. Archivos en Medicina Familiar, 11(1), 3-10.

Schröder, G., Knauerhase, A., Kundt, G. y Schober, H.C. (2012). Effects of physical therapy on quality of life in osteoporosis patients. A randomized clinical trial. Health and Quality of Life Outcomes, 10, 101.

Schurman, L., Bagur, A., Claus-Hermberg, H., Messina, O.D., Negri, A.L., Sñanchez, A., González, C., Diehl, M., Rey, P., Gamba, J., Chiarpenello, J., Moggia, M.S., Mastaglia, S. (2013). Guías 2012 para el diagnóstico, la prevención y el tratamiento de la osteoporosis. Medicina (Buenos Aires), 73, 55-74.

SEIOMM.org. Recuperado el 21 de octubre del 2013 de: http://www.seiomm.org/noticia/vistaprevia/la-fhoemo-seiomm-y-aecos-celebro-el-domingo-20-octubre-el-dia-mundial-de-laosteoporosis.

Sociedad Española de Geriatría y Gerontología (2012). Tu salud en marcha. Guía de ejercicio físico para mayores. Madrid: Grupo ICM Comunicación.

Stolzenberg, N., Belavy, D.L., Rawer, R. y Felsenberg, D. (2013). Whole-body vibration versus proprioceptive training on postural control in post-menopausal osteopenic women. Gait \& Posture, 38, 416-420.

Wayne, P.M., Kiel, D.P., Buring, J.E., Connors, E.M., Bonato, P., Yeh, G.Y., Cohen, C.J., Mancinelli, C. y Davis, R.B. (2012). Impact of Tai Chi exercise on multiple fracturerelated risk factors in post-menopausal osteopenic women: a pilot pragmatic, randomized trial. BMC Complementary and Alternative Medicine, 12, 7.

Recibido: 7 de abril de 2014 Recepción Modificaciones: 26 de mayo de 2014 Aceptado: 4 de agosto de 2014 